\title{
ENERGY SAVINGS IN SDN NETWORKS
}

\author{
Péter András Agg $^{1 *}$, Zsolt Csaba Johanyák ${ }^{2}$ \\ 1Department of Information Technology, GAMF Faculty of Engineering and Computer Science, John von \\ Neumann University, Hungary \\ https://doi.org/10.47833/2021.1.CSC.005
}

\begin{tabular}{l} 
Keywords: \\
Software-Defined Network \\
SDN energy saving \\
TCAM \\
GAL, \\
RAMD, RMAD+ \\
Article history: \\
Received April 7, 2021 \\
Revised $\quad$ April 12, 2021 \\
Accepted $\quad$ April 18, 2021 \\
\hline
\end{tabular}

\begin{abstract}
A significant advantage of Software-Defined Networks (SDNs) over traditional networks is the ability to directly control network behavior and direct communication with network elements. These options are useful and provide us with a quicker, more effective response in critical situations. Of course, they do not relieve us of the task of designing and operating the network optimally. In this paper, we present some possible solutions for optimizing SDN networks in terms of energy management.
\end{abstract}

\section{Introduction -SDN and Traditional Networks}

Nowadays the size of networks constantly increases; therefore, we need more flexible and dynamic network operations to modify the network infrastructure. It is important that the devices are easy to program and centrally controlled according to current network needs. Today, the use of SoftwareDefined Networks (SDNs) [6] is a great help to fulfill these requirements.

SDN separates the control plane and provides the data plane and logically centralized control to the network. Thus, the network devices will only be responsible for forwarding the data, while the delivery to the destination is performed based on the instructions received from the control plane. In conventional networks (Fig. 1), these two functions (data forwarding and control) are united on a single device, significantly increasing the time required for reconfiguration. In SDN networks, devices located in the data plane communicate with the controller via the Southbound API using the OpenFlow [4] protocol.

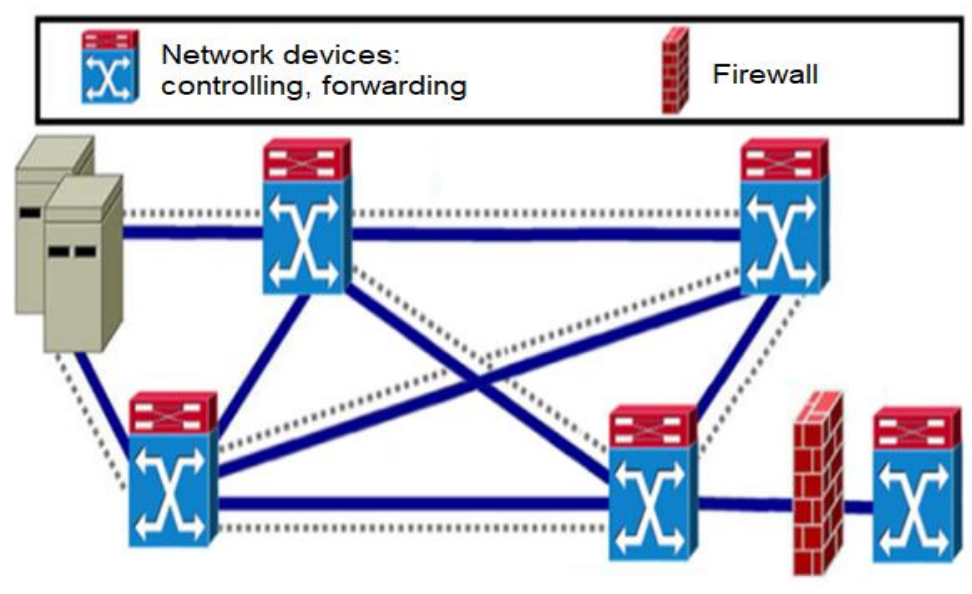

Figure 1. The architecture of the traditional networks

Separating planes allows for more efficient programmability, increases functionality, and provides the ability to remotely monitor using a single open protocol. The SDN structure allows quick response

\footnotetext{
* Corresponding author: agg.peter@gamf.uni-neumann.hu
} 
to current network needs with continuous monitoring by reconfiguring the network, which is greatly accelerated by the SDN controller. Figure 2 shows the architecture of SDN networks.

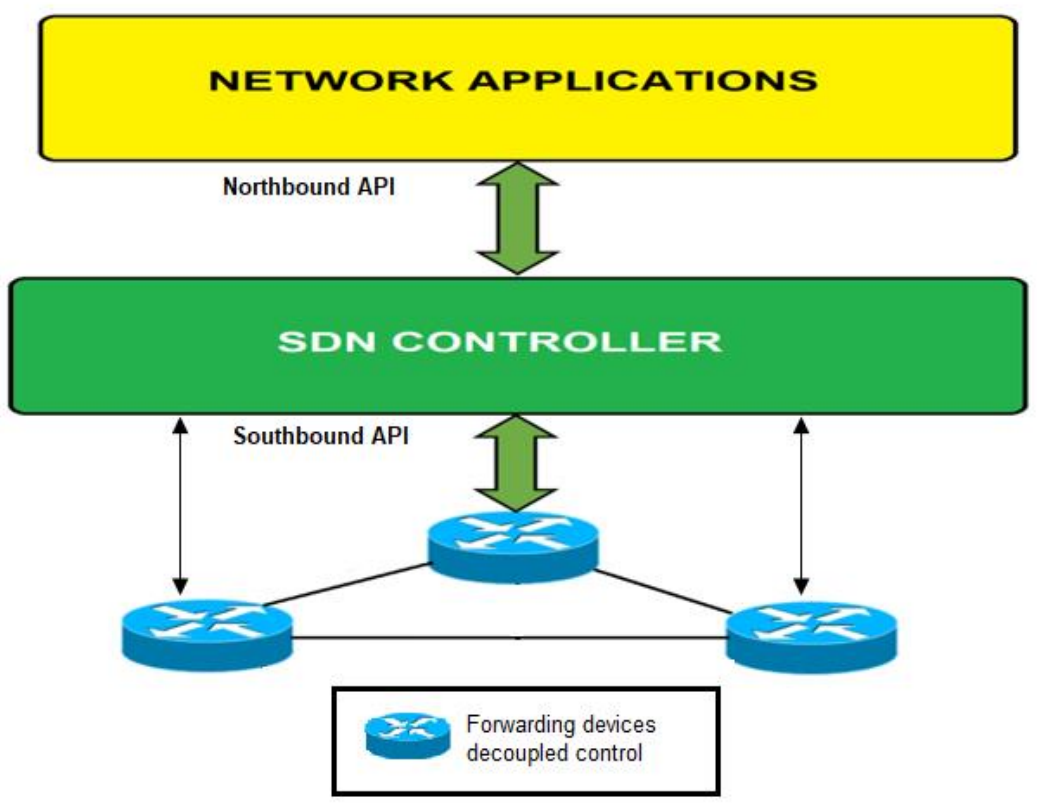

Figure 2. The architecture of the SDN networks

\section{Energy Efficiency in Computer Networks - SDN - Green Networks}

Traditional network technologies use a large amount of energy and so one cannot describe them as an environmentally or economically efficient solution. Several studies [3] analyze the carbon emission and operating costs of these networks. Although leaders in different countries are doing everything they can to reduce these parameters, the situation is still quite worrying. Therefore, rising electricity costs and rising carbon dioxide $\left(\mathrm{CO}_{2}\right)$ emissions are expected. Currently, ICT-based $\mathrm{CO}_{2}$ emissions are growing by $6 \%$ a year. This is not a good direction, so the change of model has started towards the so-called energy efficiency communication networks, aka the green networks. SDN's centralized decision-making mechanism provides an adequate opportunity to implement these networks.

Parts of SDN networks can be dynamically configured to reduce power consumption. Several possible directions have been identified to achieve this goal. One possible method is to adjust the flow according to the current traffic on the network, with proper monitoring, and quick response while putting devices not used in the network into sleep mode. Of course, if the traffic is low, it is enough to put certain unused ports to sleep instead of the entire device. The other possible direction is to optimize or possibly reduce the amount of memory used by the switches. It could lead to a significant result because the control tables required for data transmission are stored in TCAM (Ternary Content Addressable Memory), which is expensive and energy-intensive. The SDN controller can place new restrictions or new rules on switches while minimizing power consumption.

\section{SDN Approaches Aiming at Energy Efficiency}

\subsection{Green Abstraction Layer (GAL)}

The Green Abstraction Layer (GAL) [1] [5] is an SDN solution that seeks traffic-aware and energy-efficient solutions. It allows internal communication between network devices, which can be used to query data on energy used. In GAL, energy-saving states (Energy Aware States, EASs) [1] are defined by logically separate units of the network (e.g., switches, routing operations). The data is passed to the controller and uses real-time management strategies between network devices. 
The controller uses the Energy Optimizer module to calculate the best state of minimum energy consumption using the received EASs information.

The GAL consists of three phases:

- Discovery,

- Provisioning

- Monitoring

The discovery phase allows the controller to obtain information on the power consumption of each unit. This process is relatively time-consuming. The provisioning phase adjusts the state of the network nodes according to the optimal energy state of the network. This section is fast, efficient, use basic settings and ground rules whenever possible. Once the appropriate network state has been established, the monitoring phase continuously sends information about the current state of each entity, and the process restarts if necessary.

\subsection{Exclusive Routing (EXR) - Fair-Sharing Routing (FSR)}

Exclusive Routing (EXR) [9] can be a useful method for energy saving. EXR is an improved version of Fair-Sharing Routing (FSR) [9]. FSR selects a subset of the links and distributes the flows evenly without delay. This is a useful method because the traffic is distributed evenly, the switches are not overloaded. However, this method uses only about $55 \%$ of the capacity of the switches. This ratio is not adequate from the energy consumption point of view which led to the development of the EXR method. The main task of EXR is to eliminate the low utilization of the links and the switches.

EXR tries to maximize the use of the capacity of the active links and to turn off the unused links to save more energy. EXR allows the definition of the flow priorities depending on the size of the flow or the flow deadline. EXR has two kinds of flows, the suspended and the active ones. When a new flow arrives, the controller notifies the switches what to do. They can block or let the flow through. If there is a path without any flows, the controller sets the flow as active and sends it. If there is no such route, it checks to see if there is a path whose suspended flow has lower priorities than the current flow. If there is such one then it is forwarded on this path. If the option outlined above does not exist, it will suspend the flow. If more than one of the listed options exists, the number of free switches determines the flow path. When the selection process is complete, the controller sends the necessary information to the switches. The results show that this method can achieve near $100 \%$ capacitance utilization, so we can save significant energy.

\subsection{Routing for Minimization of Active Devices (RMAD, RMAD+)}

Another possible SDN-based energy-saving solution is the so-called Routing for Minimization of Active Devices (RMAD) [7]. This method basically follows the OSPF (Open Shortest Path First) strategy but takes into account the energy saved when turning devices on and off. The RMAD practically finds the shortest route with the most active links. It tries not to activate another link, thus saving energy as it increases the sleep time of already dormant connections.

The enhanced version of RMAD (called RMAD+) [7] limits the number of hops on the route and will select the route with the fewest active nodes. The reason for this technique is that the nodes consume more energy than connections (links), so this method can save more energy. It is important to note that RMAD+ chooses the path with the fewest active nodes, which is not necessarily the shortest path.

\subsection{Rule Placement- and TCAM-based Energy-aware SDN Approach}

This method is practically used to eliminate the problem of energy-aware routing (EAR) rule space [2]. According to the EAR approach, there is an infinite number of rule areas, i.e. the OpenFlow switch can store an infinite number of rules. Of course, this is not true in practice; the TCAM (switch memory) has limited storage space which is expensive and energy-intensive, which is not necessarily useful in reducing costs. Therefore, a solution is necessary for this problem. 
There is a default rule, which has the lowest priority and takes effect when there is no predefined rule for a packet, i.e. it is not known how to forward it. Communication between network devices and the controller is time-consuming, delayed, and consumes much power. To eliminate these costs, packets are processed by the default rule and are forwarded to the default port without contacting the controller, which saves significant time. Each switch must have only one default port.

To minimize energy consumption, a heuristic algorithm was proposed in [2] for large networks. The algorithm first finds the appropriate route for all incoming requests. This algorithm takes care of the capacity on links and rule area constraints. With the specified paths, the direction board is loaded until the memory is full. The port that participated in most management tasks will be set as the default port, so there will be more space on multiple ports to receive the new rules. It is important to note that this method reduces the number of active links. The links with low traffic are placed in sleep mode and their traffic is transferred to other active connections (even the default port). With this method, quite a lot of energy can be saved. Using this algorithm, the size of the rule area is reduced, the communication delay between the devices and the controller is also reduced, and the total number of active connections will decrease.

\subsection{Virtual Machine Placement-based Energy-aware SDN Approach}

Traffic-aware placement of Virtual Machines (VMs) in data center networks (DCNs) can also significantly contribute to better utilization of SDN resources. There are two approaches to accomplish this task: server-driven and network-driven, respectively. These algorithms aim the efficient intra-DCN resource allocation. The server-driven algorithm first chooses a server for a VM and then determines the switches that will provide the flow of traffic. The network-driven algorithm first selects the switches and then the server for the VM.

There are three options to select the servers and the switches:

- $\quad$ First Fit (FF)

- Best Fit (BF)

- Worst Fit (WF).

The FF method ranks servers and switches in ascending order according to their computational power and overall traffic load and then chooses the first one from the list. WF chooses only the most unloaded servers or switches. BF uses the most loaded servers or switches. Results show that the network-driven algorithm with the FF method saves the most energy [8].

\section{Conclusion - Challenges}

The presented SDN-based energy saving solutions can be used well in practice. However, the comparison of the different solutions based only on the literature is difficult because the papers proposing them published only results of tests carried out with special parameter values, topologies, network conditions, and simulation software, which were different in each case. A quantitative comparison of their efficiency in terms of energy savings can be carried out only by implementing them on the same platform. Table 1 shows a qualitative evaluation the efficiency of the presented methods based on literature review.

The table shows that one can achieve the most efficient energy savings with heuristic rule space modification (taking into account the level of implementation) according to the limitations of wired networks.

An important milestone for future research could be rule placement, which directly affects routing and thus network performance and energy efficiency. The SDN controller must place energysaving, adaptive rules in the switches with certain restrictions that increase energy efficiency. Further research will be focused on the investigation of the applicability of different fuzzy techniques (e.g. [10][11][12][13]) for the improvement of energy savings.

We also consider the need for security as important. Communication of control data and control applications can face threats. Their secure communication requires further development as well. 
Table 1. Energy saving SDN solutions and their key features

\begin{tabular}{|c|c|c|c|c|c|}
\hline Protocol name & $\begin{array}{c}\text { Used } \\
\text { parameters }\end{array}$ & Used method & $\begin{array}{l}\text { Energy } \\
\text { efficiency } \\
\text { level }\end{array}$ & $\begin{array}{l}\text { Difficulty of the } \\
\text { implementation }\end{array}$ & $\begin{array}{c}\text { Target } \\
\text { environment }\end{array}$ \\
\hline GAL & $\begin{array}{c}\text { Energy aware } \\
\text { states, green } \\
\text { abstraction } \\
\text { layer, power } \\
\text { management }\end{array}$ & Traffic-aware & High & $\begin{array}{l}\text { Hard to } \\
\text { implement }\end{array}$ & Fixed Network \\
\hline RMAD & $\begin{array}{l}\text { Sleep ratio, } \\
\text { number of } \\
\text { active nodes }\end{array}$ & Traffic-aware & Medium & $\begin{array}{l}\text { Easy to } \\
\text { implement }\end{array}$ & Fixed Network \\
\hline $\begin{array}{l}\text { Heuristic } \\
\text { algorithm rule- } \\
\text { placement }\end{array}$ & $\begin{array}{l}\text { TCAM, Rule } \\
\text { placement }\end{array}$ & $\begin{array}{l}\text { Rule space- } \\
\text { based }\end{array}$ & High & $\begin{array}{l}\text { Easy to } \\
\text { implement }\end{array}$ & $\begin{array}{l}\text { Data Center } \\
\text { Networks, } \\
\text { Fixed } \\
\text { Networks }\end{array}$ \\
\hline $\begin{array}{l}\text { NV-aware } \\
\text { traffic energy } \\
\text { save }\end{array}$ & Link utilization & $\begin{array}{c}\text { Network } \\
\text { Virtualization }\end{array}$ & Low & $\begin{array}{l}\text { Hard to } \\
\text { implement }\end{array}$ & $\begin{array}{l}\text { Data Center } \\
\text { Networks }\end{array}$ \\
\hline $\begin{array}{c}\text { Exclusive } \\
\text { Routing }\end{array}$ & $\begin{array}{l}\text { Active and } \\
\text { suspended } \\
\text { flows }\end{array}$ & Traffic-aware & Medium & $\begin{array}{l}\text { Easy to } \\
\text { implement }\end{array}$ & Fixed Network \\
\hline
\end{tabular}

\section{Acknowledgment}

This research is supported by EFOP-3.6.1-16-2016-00006 "The development and enhancement of the research potential at John von Neumann University" project. The Project is supported by the Hungarian Government and co-financed by the European Social Fund.

\section{References}

[1] R. Bolla , T. Szemethy , R. Bruschi , F. Davoli , L. Di Gregorio , P. Donadio, et al., The green abstraction layer: a standard power-management interface for next-generation network devices, IEEE Internet Comput. (2013) 82-86. DOI: $10.1109 / \mathrm{MIC} .2013 .39$

[2] F. Giroire , J. Moulierac , T.K. Phan, Optimizing rule placement in software-de- fined networks for energy-aware routing, Global Communications Conference (GLOBECOM), 2014 IEEE, 2014, pp. $2523-2529$. DOI: $10.1109 /$ GLOCOM.2014.7037187

[3] T.C. Group, (2010). Smart 2020 - enabling the low carbon economy in the in- formation age. Available: https://gesi.org/research/download/7 [Retrieved 05-03-2021].

[4] A. Lara, A. Kolasani, and B. Ramamurthy, Network innovation using OpenFlow: A survey, IEEE Communications Survey \& Tutorials, Vol. 16, NO. 1. First quarter 2014 493-512. DOI: $10.1109 /$ SURV.2013.081313.00105

[5] D. Li , Y. Shang, C. Chen, Software defined green data center network with exclusive routing, in: INFOCOM, 2014 Proceedings IEEE, 2014, pp. 1743-1751. DOI: $10.1109 /$ INFOCOM.2014.6848112

[6] Diego Kreutz, Fernando M. V. Ramos, Paulo Verissimo, Software-Defined Networking: A Comprehensive Survey, Proceedings of the IEEE, Vol. 103, No. 1, January 2015 pp. 14-76. DOI: $10.1109 /$ JPROC.2014.2371999

[7] Mehmet Fatih Tuysuz, Zekiye Kubra Ankarali , Didem Gözüpek, A survey on energy efficiency in software defined networks, Computer Networks 113, 2017 188-204.

DOI: $10.1016 /$ i.comnet.2016.12.012

[8] M. Gharbaoui , B. Martini , D. Adami , G. Antichi , S. Giordano , P. Castoldi , On vir tualization-aware traffic engineering in OpenFlow data centers networks, in: Network Operations and Management Symposium (NOMS), 2014 IEEE, 2014, pp. 1-8.

DOI: $10.1109 /$ NOMS.2014.6838318

[9] D. Li , Y. Shang, C. Chen, Software defined green data center network with exclusive routing, in: INFOCOM, 2014 Proceedings IEEE, 2014, pp. 1743-1751.

DOI: $10.1109 /$ INFOCOM.2014.6848112 
[10] R.E. Precup and M.L. Tomescu, Stable fuzzy logic control of a general class of chaotic systems, Neural Computing and Applications, vol. 26, no. 3, pp. 541-550, Apr. 2015. DOI: $10.1007 / \mathrm{s} 00521-014-1644-7$

[11] I. Škrjanc and S. Blažič (2005): Predictive functional control based on fuzzy model: design and stability study, Journal of Intelligent and Robotic Systems, vol. 43, no. 2-4, pp. 283-299, Aug. 2005. DOI: $10.1007 / \mathrm{s} 10846-005-5138-9$

[12] J. Vaščák and M. Rutrich, Path planning in dynamic environment using fuzzy cognitive maps, Proceedings of 6th International Symposium on Applied Machine Intelligence and Informatics (SAMI 2008), Herlany, Slovakia, 2008, pp. 5-9.

[13] T. Tompa, Sz. Kovács, D. Vincze, M. Niitsuma: Demonstration of expert knowledge injection in Fuzzy Rule Interpolation based Q-learning, In: 2021 IEEE/SICE International Symposium on System Integration (SII) (2021) pp. 843-844. , 2 p.

DOI: $10.1109 /$ IEEECONF49454.2021.9382734 